\section{Short-Pulse Electromagnetic Scattering from Arbitrarily Oriented Subsurface Ordnance}

\author{
Norbert Geng and Lawrence Carin
}

\begin{abstract}
A rigorous method-of-moments (MoM) analysis is used to model wide-band scattering from general three-dimensional perfectly conducting objects buried in a lossy layered medium. Here, we focus on ordnance buried in a half space (soil). The time-domain fields scattered from a tilted antitank mine are examined in detail as a function of polarization and observation position.
\end{abstract}

Index Terms-Radar, scattering, short-pulse.

\section{INTRODUCTION}

The method of moments (MoM) has been used as a modeling tool for nearly three decades [1], and over this time, it has been used to analyze a wide range of scattering problems. Here, we are interested in wide-band (short-pulse) scattering from three-dimensional buried ordinance with application to the detection of buried mines and unexploded ordnance (UXO) [2]-[9]. For such problems, one requires the dyadic layered-medium Green's function [2], [3], [6]-[9], which is often specialized to the half-space case. This Green's function has been discussed in detail in [10], where several alternative formulations have been presented. For wide-band applications, one must efficiently evaluate the Sommerfeld-type integrals required for computation of the dyadic Green's function [10]. Here, we utilize the method of complex images, a technique developed by Chow et al. [11], [12] several years ago, and recently extended for general three-dimensional targets [13].

Therefore, the techniques used here to analyze scattering from a general buried perfectly conducting target are not new, the triangularpatch MoM formulation [1], [14], [15], layered-medium Green's function [10], and complex-image technique [11]-[13] having been published elsewhere. However, despite the importance of the buriedordnance problem, there are few results published in the literature on the phenomenology underlying this application. We seek to fill this void, considering an antitank mine oriented arbitrarily, with shortpulse excitation of general polarization. Previous MoM results for this problem [2], [3], [6]-[9] considered both perfectly conducting and dielectric (plastic) mines, but the problem was simplified by assuming that the mine-(layered medium) composite was a body of revolution (BOR). This BOR assumption requires that the axis of the target be perpendicular to the air-ground interface, precluding an examination of the importance of the mine tilt on the scattered fields.

One can also model scattering from general buried targets through use of the finite-difference time-domain (FDTD) method [4]. Previous FDTD results have concentrated on near-field applications, in which interactions between the antenna and target are accounted for rigorously [4]. Here, we are interested in applications for which the sensor is distant from the target (synthetic aperture radar [6], [7]) which, in principle, could also be analyzed via FDTD. We utilize

Manuscript received August 12, 1998; revised October 13, 1998.

N. Geng was with the Department of Electrical and Computer Engineering, Duke University, Durham, NC 27708-0291 USA. He is now with the Institüt für Hoechstfrequenztechnik und Elektronik, University of Karlsruhe, D-76128 Karlsruhe, Germany.

L. Carin is with the Department of Electrical and Computer Engineering, Duke University, Durham, NC 27708-0291.

Publisher Item Identifier S 0196-2892(99)03487-7. the MoM in lieu of FDTD because the former only requires spatial discretization on the target surface (for a surface MoM formulation [14], [15]), while the latter necessitates discretization of the entire computational domain.

Our principal focus here is on the presentation of example results, which will serve two purposes. First, the detailed phenomenological study will shed light on the physics underlying wide-band scattering from buried ordnance. Second, since, to our knowledge, similar results have not been published previously, the data presented here will be of interest for comparison to future computational algorithms, be they integral-equation (MoM) or differential-equation (FDTD) based.

\section{Phenomenological Study}

Consider a cylindrical perfectly conducting target, of $33 \mathrm{~cm}$ diameter and $16 \mathrm{~cm}$ height, buried in soil with dielectric constant $\epsilon_{r}=4$ and conductivity $\sigma=0.005 \mathrm{~S} / \mathrm{m}$. We have performed detailed analyzes on the effects of soil type on the scattered fields, and the soil properties selected here are representative of a wide class of soils [7], [16]. The MoM analysis can readily handle dispersive soils [2], [3], [6]-[9], although here our focus is on the target itself. The cylindrical target selected closely resembles the M20 antitank mine [6], [7]. Finally, the computer code was validated through comparison with published results [2], [3], [7], in which the special case of nontilted bodies of revolution (BOR's) was considered.

It has been shown in a previous correspondence [6] that the crosspolarized fields backscattered from a BOR are zero, and this has been validated experimentally through consideration of the nontilted M20 mine [6]. Since many forms of anthropic clutter do not satisfy the BOR model, the cross-polarized fields potentially provide a powerful discriminant for distinction of targets and clutter [6]. However, for a buried target, the BOR model is only appropriate if the target axis is perpendicular to the air-ground interface, such that the target-(layered medium) composite satisfies the BOR model [6]. In practice, however, one may anticipate that the buried mine may be tilted, such that the BOR-composite model is no longer appropriate. It is of interest to determine the degree to which this phenomenon corrupts the ideal properties of the cross-polarized scattered fields.

A second issue of interest involves the aspect-dependent nature of the fields scattered from a buried tilted mine. If the target-(half space) composite satisfies the BOR model, the scattered fields are only dependent on the spherical coordinates $(r, \theta)$, independent of the azimuthal position $\phi$, assuming that the center of the coordinate system is along the BOR axis. Now, consider a tilted mine, with the center of the coordinate system at the center of the mine, and with the $z$ axis defined as perpendicular to the air-ground interface. If the backscattered fields from the tilted mine are a strong function of the azimuthal angle $\phi$, then the difficulty of target detection is enhanced significantly. Moreover, for synthetic aperture radar (SAR) applications [6], [7], the scattered fields from multiple target-sensor orientations are employed to generate a SAR image. Consequently, a strong dependence of the tilted-mine backscattered fields on the target-sensor orientation will have an important effect on the subsequent SAR imagery.

We first consider the polarimetric issue, and in Fig. 1 plot the $H V$ (horizontally polarized receive fields, and vertically polarized excitation fields) for a mine tilted $5^{\circ}$. A pulsed plane wave is assumed incident $30^{\circ}$ from grazing, with a pulse shape as shown in Fig. 1; the incident pulse has a center frequency of $500 \mathrm{MHz}$ and 


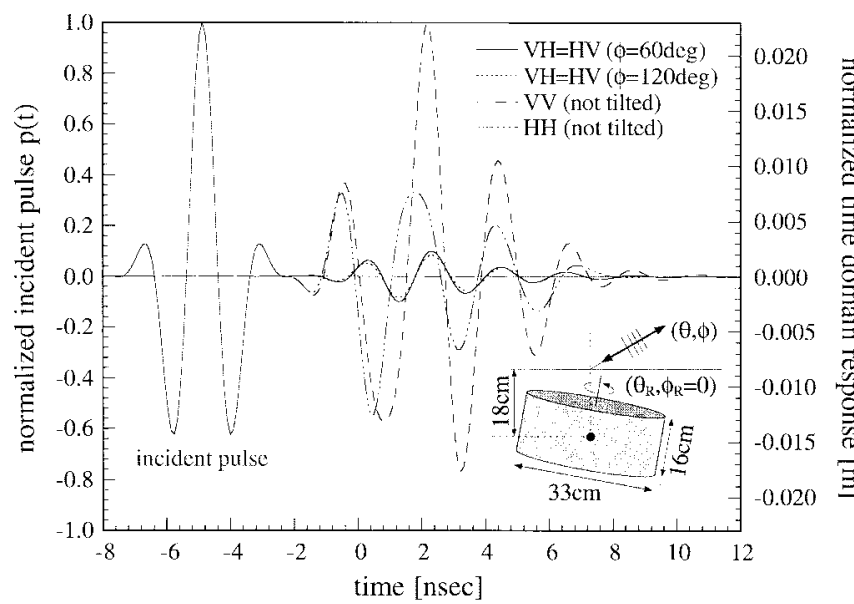

Fig. 1. Time-domain fields backscattered from a tilted perfectly conducting cylinder, which simulates an M20 antitank mine (inset). We consider the target tilted at $5^{\circ}$. Shown are the incidentpulse (scale on left) and four normalized backscattered waveforms (scale on right). The cross-polarized $H V$ fields (which are the same as the $V H$ fields) are shown for azimuthal angles $\phi=60^{\circ}$ and $\phi=120^{\circ}$. For comparison, results are also shown for the copolarized $V V$ and $H H$ fields for the casewhen the mine is not tilted. The soil has $\epsilon_{r}=4$ and $\sigma=0.005 \mathrm{~S} / \mathrm{m}$, and the excitation plane wave is incident at $30^{\circ}$ from grazing.

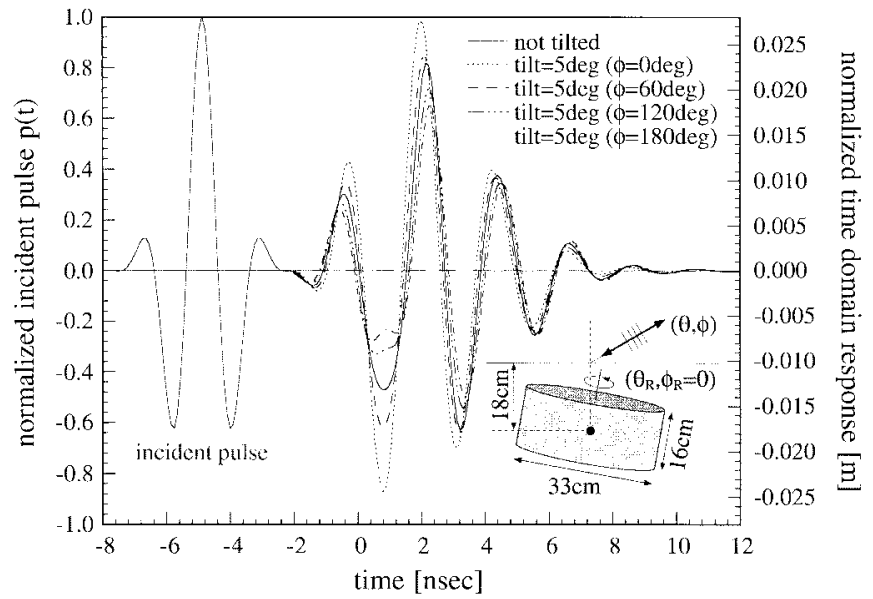

Fig. 2. As in Fig. 1, but for $V V$ polarization. Results are shown for $\phi=0^{\circ}, 60^{\circ}, 120^{\circ}$, and $180^{\circ}$. Backscattered fields are also shown for the case when the mine is not tilted, for which the scattered fields are independent of $\phi$.

significant frequencies that extend to approximately $1 \mathrm{GHz}$. The farzone, cross-polarized backscattered fields are plotted for azimuthal angles $\phi=60^{\circ}$ and $\phi=120^{\circ}$, where $\phi=0^{\circ}$ defines a bisectional symmetry plane through the tilted target. Note that the cross-polarized backscattered fields vanish if the wave vector is in the $\phi=0^{\circ}$ plane [6]. To quantify the relative strength of the cross-polarized backscattered fields, we also plot the $V V$ and $H H$ backscattered fields for the case when the mine is not tilted (for which case, as mentioned, there are no cross-polarized fields). Each backscattered far-zone time-domain waveform is normalized through multiplication by the observation distance $r$ (each waveform is also time shifted). We see from Fig. 1 that the tilted mine yields appreciable crosspolarized backscattered fields, even for the relatively slight $\left(5^{\circ}\right)$ tilt considered here. Although not shown due to space constraints, we also considered the same mine tilted $10^{\circ}$, for which we witnessed an approximate doubling of the backscattered $H V$ response. These results demonstrate that the cross-polarized fields for a mine-which

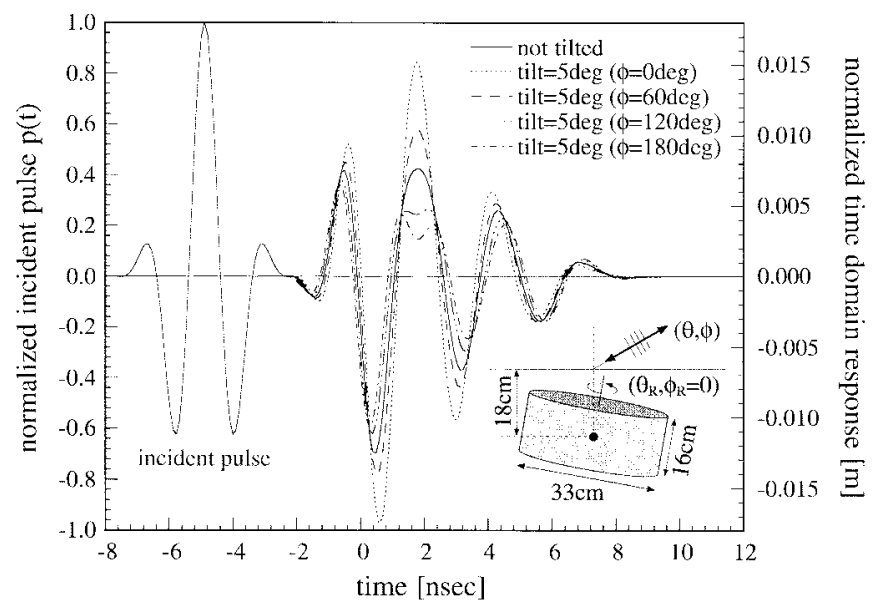

Fig. 3. As in Fig. 2, $H H$ polarization.

are ideally zero if the mine is oriented with its axis perpendicular to the soil-are appreciable even for a modest tilt. This may vitiate the utility of polarimetry for SAR-based target identification. A more conclusive assessment of this issue will require examination of the relative strength of cross-polarized fields from clutter of interest.

We now consider the copolarized fields scattered from a tilted mine as a function of the azimuthal angle $\phi$. In Fig. 2, we consider $V V$ backscattering, for a $5^{\circ}$ mine tilt, for angles $\phi=0^{\circ}, 60^{\circ}, 120^{\circ}$, and $180^{\circ}$. For comparison, we also consider the case when the mine is not tilted, for which the backscattered fields are the same for all $\phi$. For the $\phi=0^{\circ}$ case, the mine is tilted forward toward the sensor, yielding enhanced backscattered signal strength relative to the case of a nontilted mine. Moreover, we see that the backscattered fields are a strong function of the angle $\phi$, complicating target identification. Although the detailed shape of the backscattered fields are different for $H H$ polarization (Fig. 3), these are again a strong function of the target-sensor orientation $(\phi)$. Space limitations preclude showing results for the case of a $10^{\circ}$ tilt, but this case again manifests an even stronger difference in the backscattered fields relative to the nontilted mine. In fact, at $\phi=0^{\circ}$, the backscattered $V V$ fields were almost twice the strength of the fields for the nontilted case, with similar effects witnessed for $H H$ polarization.

\section{CONCLUSION}

A rigorous MoM analysis has been performed on the polarmetric time-domain fields scattered from a tilted antitank mine buried in soil. The mine may initially be placed in the ground in a tilted manner, or such a tilt could be caused by movement of the mine after being in the ground through multiple seasons (many mines have been in the ground for decades). Our results demonstrate that, even for a relatively modest tilt of $5^{\circ}$, the backscattered fields are significantly altered relative to the no-tilt case. In particular, if not tilted, BOR's have no cross-polarized fields. However, here we have shown that tilted mines have significant cross-polarized fields, even for a relatively small tilt angle. Moreover, for the tilted mine, the $V V$ and $H H$ scattered fields are a strong function of the target-sensor orientation, complicating target identification and SAR imaging. However, these results also underscore the benefit of rigorous modeling, with which such phenomena can be predicted a priori and exploited (or, at least, accounted for) in the context of signal processing. Finally, we note that the dependence of the backscattered fields on the tilt angle is a function of the system bandwidth. For more narrow bandwidth systems than considered here, the dependence on the tilt angle will be less appreciable. However, 
in this case, the target appears as a point scatterer, for which little discrimination capability is possible.

\section{REFERENCES}

[1] R. F. Harrington, Field Computation by Moment Methods. New York: IEEE Press, 1993.

[2] S. Vitebskiy, K. Sturgess, and L. Carin, "Short-pulse plane-wave scattering from buried perfectly conducting bodies of revolution," IEEE Trans. Antennas Propagat., vol. 44, pp. 143-151, Feb. 1996.

[3] S. Vitebskiy and L. Carin, "Resonances of perfectly conducting wires and bodies of revolution buried in a lossy dispersive half-space," IEEE Trans. Antennas Propagat., vol. 44, pp. 1575-1583, Dec. 1996.

[4] J. M. Bourgeois and G. S. Smith, "A fully three-dimensional simulation of a ground-penetrating radar: FDTD theory compared with experiment," IEEE Trans. Geosci. Remote Sensing, vol. 34, pp. 36-44, Jan. 1996.

[5] L. Peters, Jr., J. J. Daniels, and J. D. Young, "Ground penetrating radar as a subsurface environmental sensing tool," Proc. IEEE, vol. 82, pp. 1802-1822, Dec. 1994

[6] L. Carin, R. Kapoor, and C. E. Baum, "Polarimetric SAR imaging of buried landmines," IEEE Trans. Geosci. Remote Sensing, vol. 36, pp. 1985-1988, Nov. 1998.

[7] S. Vitebskiy, L. Carin, M. A. Ressler, and F. H. Le, "Ultra-wideband, short-pulse ground-penetrating radar: Simulation and measurement," IEEE Trans. Geosci. Remote Sensing, vol. 35, pp. 762-772, May 1997.

[8] N. Geng, D. Jackson, and L. Carin, "On the resonances of buried dielectric bodies of revolution," IEEE Trans. Antennas Propagat., submitted for publication.

[9] N. Geng and L. Carin, "Short-pulse scattering from buried dielectric bodies of revolution," IEEE Trans. Antennas Propagat., to be published.

[10] K. A. Michalski and D. Zheng, "Electromagnetic scattering and radiation by surfaces of arbitrary shape in layered media, Part I: Theory," IEEE Trans. Antennas Propagat., vol. 38, pp. 335-344, Mar. 1990.

[11] Y. L. Chow, J. J. Yang, D. G. Fang, and G. E. Howard, "A closed-form spatial Green's function for the thick microstrip substrate," IEEE Trans. Microwave Theory Tech., vol. 39, pp. 588-592, Mar. 1991.

[12] R. M. Shubair and Y. L. Chow, "A simple and accurate complex image interpretation of vertical antennas present in contiguous dielectric halfspaces," IEEE Trans. Antennas Propagat., vol. 41, pp. 806-812, June 1993.

[13] M. I. Aksun, "A robust approach for the derivation of closed-form Green's functions," IEEE Trans. Microwave Theory Tech., vol. 44, pp. 651-658, May 1996.

[14] S. M. Rao, D. R. Wilton, and A. W. Glisson, "Electromagnetic scattering from surfaces of arbitrary shape," IEEE Trans. Antennas Propagat., vol. AP-30, pp. 409-418, May 1982.

[15] D. R. Wilton, S. M. Rao, A. W. Glisson, D. H. Schaubert, O. M. AlBundak, and C. M. Butler, "Potential integrals for uniform and linear source distributions on polygonal and polyhedral domains," IEEE Trans. Antennas Propagat., vol. 32, pp. 276-281, Mar. 1984.

[16] J. E. Hipp, "Soil electromagnetic parameters as functions of frequency, soil density, and soil moisture," Proc. IEEE, vol. 62, pp. 98-103, Jan. 1974.

\section{Covariance Estimation with Limited Training Samples}

Saldju Tadjudin and David A. Landgrebe

Abstract - This paper describes a covariance estimator formulated under an empirical Bayesian setting to mitigate the problem of limited training samples in the Gaussian maximum likelihood (ML) classification for remote sensing. The most suitable covariance mixture is selected by maximizing the average leave-one-out log likelihood. Experimental results using AVIRIS data are presented.

Index Terms - Covariance estimation, Gaussian maximum likelihood, leave-one-out log likelihood, regularization.

\section{INTRODUCTION}

In the conventional Gaussian maximum likelihood (ML) classifier, the classification rule can be expressed in the form of a discriminant function and a sample is assigned to the class with the largest discriminant function value. A multivariate Gaussian density function is given as

$$
\begin{gathered}
f_{i}(x)=(2 \pi)^{-p / 2}\left|\Sigma_{i}\right|^{-1 / 2} \exp \left[-\frac{1}{2}\left(x-\mu_{i}\right)^{T} \Sigma_{i}^{-1}\left(x-\mu_{i}\right)\right] \\
1 \leq i \leq L
\end{gathered}
$$

where $x \in \Re^{p}, \mu_{i}$, and $\Sigma_{i}$ are the $i$ th class mean vector and covariance matrix, respectively, and $L$ is the number of classes. Assuming a $[0,1]$ loss function, the ML classification rule then becomes

$$
d_{i}(x)=\min _{i \leq i \leq L} d_{i}(x)
$$

where $d_{i}$ is the discriminant function given by

$$
d_{i}(x)=\left(x-\mu_{i}\right)^{T} \Sigma_{i}^{-1}\left(x-\mu_{i}\right)+\ln \left|\Sigma_{i}\right| .
$$

This classification rule is also called a quadratic classifier. A special case occurs when all of the class covariance matrices are identical. It then becomes a linear classifier

$$
\Sigma_{i}=\Sigma, \quad 1 \leq i \leq L
$$

In practical situations, the true class distributions are rarely known. Therefore, the sample estimates are computed from the training samples.

The quadratic classifier's performance can be degraded when the number of dimensions is large compared to the training set size due to the instability of sample estimates. In particular, the sample covariance estimate becomes highly variable and may even be singular. One way to deal with the instability of covariance estimate is to employ the linear classifier. By replacing each class covariance estimate with their average, leading to the linear classifier, the number

Manuscript received July 31, 1998; revised October 28, 1998. This work was supported in part by NASA under Grant NAG5-3975 and the Army Research Office under Grant DAAH04-96-1-0444.

The authors are with the School of Electrical and Computer Engineering, Purdue University, West Lafayette, IN 47907-1285 USA (e-mail: landgreb@ecn.purdue.edu).

Publisher Item Identifier S 0196-2892(99)04334-X. 